This document is confidential and is proprietary to the American Chemical Society and its authors. Do not copy or disclose without written permission. If you have received this item in error, notify the sender and delete all copies.

\title{
Effects of cationic species in salts on the electrical conductivity of doped PEDOT:PSS films
}

\begin{tabular}{|r|l|}
\hline Journal: & ACS Applied Polymer Materials \\
\hline Manuscript ID & Draft \\
\hline Manuscript Type: & Letter \\
\hline Date Submitted by the & n/a \\
\hline Complete List of Authors: & $\begin{array}{l}\text { Li, Xin; Sun Yat-Sen University, School of Materials Science and } \\
\text { Engineering, PCFM Lab } \\
\text { Liu, Zhen; Sun Yat-Sen University } \\
\text { Zhou, Zekun; Sun Yat-Sen University, School of Materials Science and } \\
\text { Engineering } \\
\text { Gao, Haiyang; Sun Yat-Sen University, School of Materials Science and } \\
\text { Engineering } \\
\text { Liang, Guo Dong; Sun Yat-Sen University, PCFM and GDHPPC labs, } \\
\text { School of Materials Science and Engineering } \\
\text { Rauber, Daniel; Universitat des Saarlandes, Chemistry } \\
\text { Kay, Christopher; Saarland University } \\
\text { Zhang, Peng; Sun Yat-Sen University, School of Materials Science and } \\
\text { Engineering }\end{array}$ \\
\hline
\end{tabular}

\section{SCHOLARONE Manuscripts}



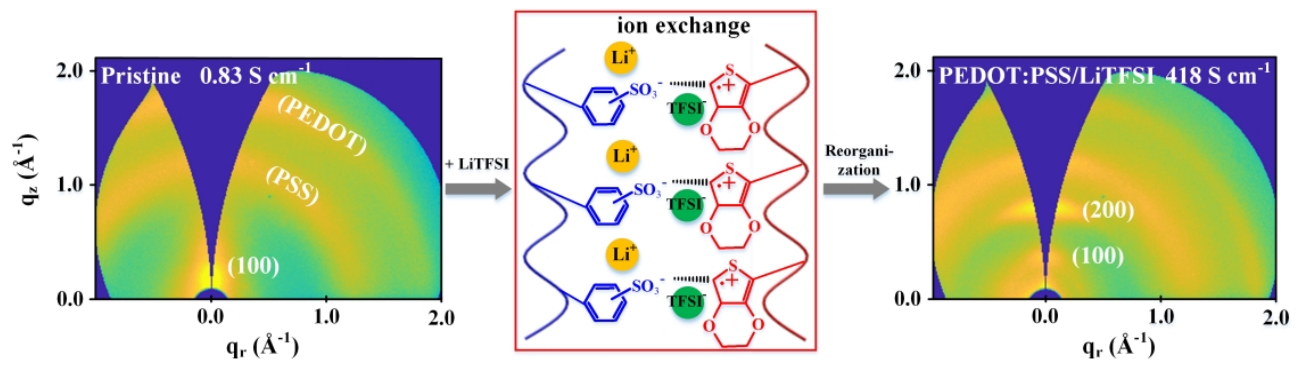

Abstract Graphic $341 \times 96 \mathrm{~mm}(300 \times 300$ DPI) 


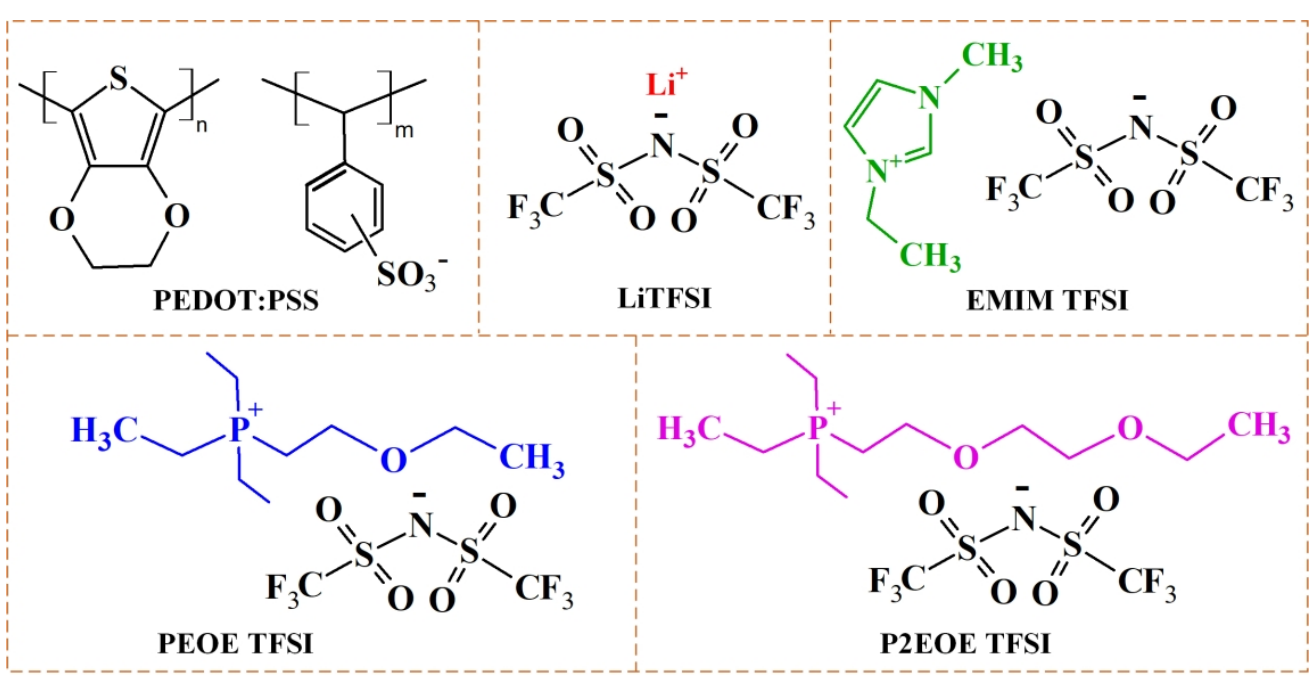

Figure 1. Chemical structure of PEDOT:PSS and ionic salts used in this work.

$202 \times 101 \mathrm{~mm}(300 \times 300$ DPI $)$ 

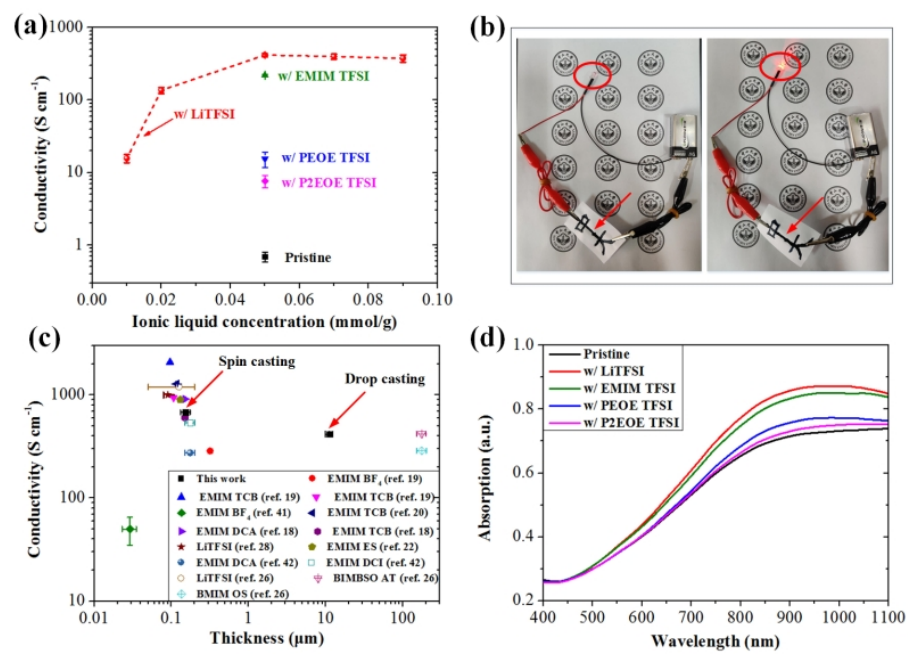

Figure 2. (a) The electrical conductivity result of PEDOT:PSS films doped with different ILs and LiTFSI at a series of concentration. (b) Photo images of LED electrical circuits and the parts for PEDOT:PSS composite deposited on paper are marked with the arrows (left: PEODT:PSS, right: PEDOT:PSS/LiTFSI composite). (c) Thickness influence on the conductivity of PEDOT:PSS film (our work is marked by the arrows). (d) UV-Vis absorption spectra of pristine PEDOT:PSS and PEDOT:PSS/IL dispersion. Notes: PEDOT:PSS/salt is denoted as $\mathrm{w} / \mathrm{salt}, \mathrm{mmol} / \mathrm{g}$ represents the stoichiometry of salts in $1.0 \mathrm{~g}$ of PEDOT: PSS water dispersion.

$$
202 \times 101 \mathrm{~mm}(300 \times 300 \text { DPI) }
$$



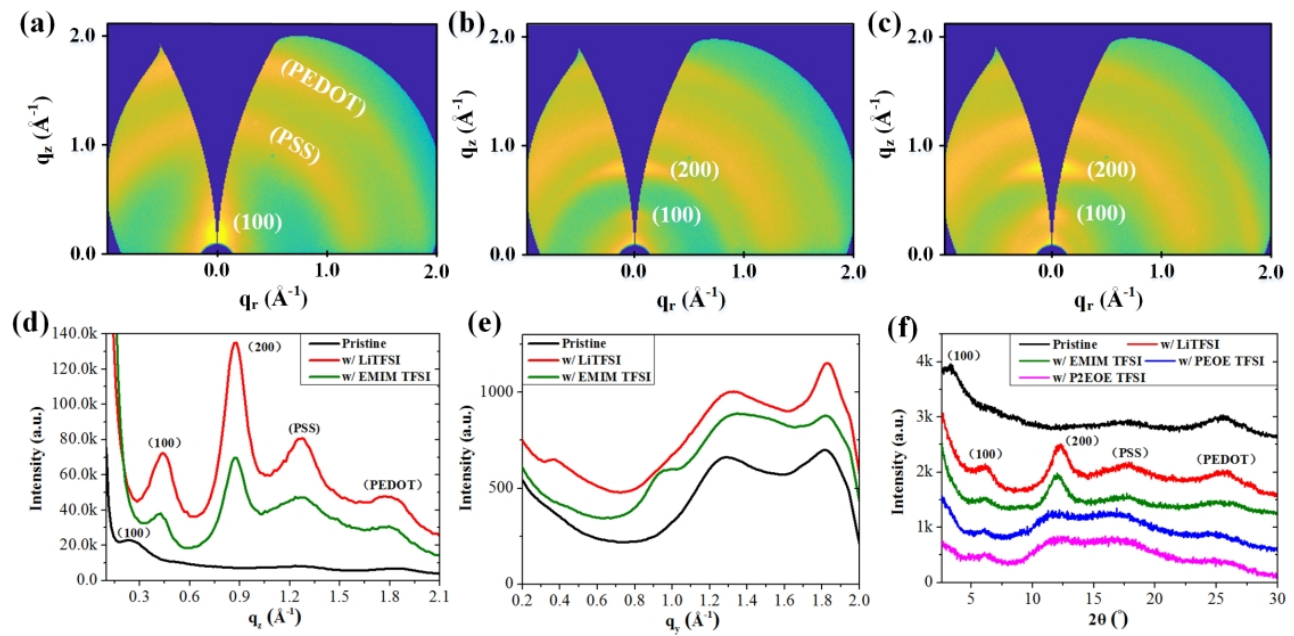

Figure 3. Typical two-dimensional GIWAXS patterns of (a) pristine, (b) EMIM TFSI-treated and (c) LiTFSItreated PEDOT:PSS films. (d) Vertical cake cuts of the initial GIWAXS data of pristine and salt-treated PEDOT:PSS films. (e) Horizontal cake cuts of the initial GIWAXS data of pristine and salt-treated PEDOT:PSS films. (f) GIXRD data of pristine and salt-treated PEDOT:PSS films. Note: PEDOT:PSS/salt is denoted as w/salt.

$202 \times 101 \mathrm{~mm}(300 \times 300 \mathrm{DPI})$ 
Figure 4. Schematic illustration of the structure of polymer composite film: loose lamellar packing of pristine PEDOT:PSS (left) and compacted lamellar packing of PEDOT:PSS /LiTFSI composite (right).

$202 \times 101 \mathrm{~mm}(300 \times 300$ DPI $)$ 

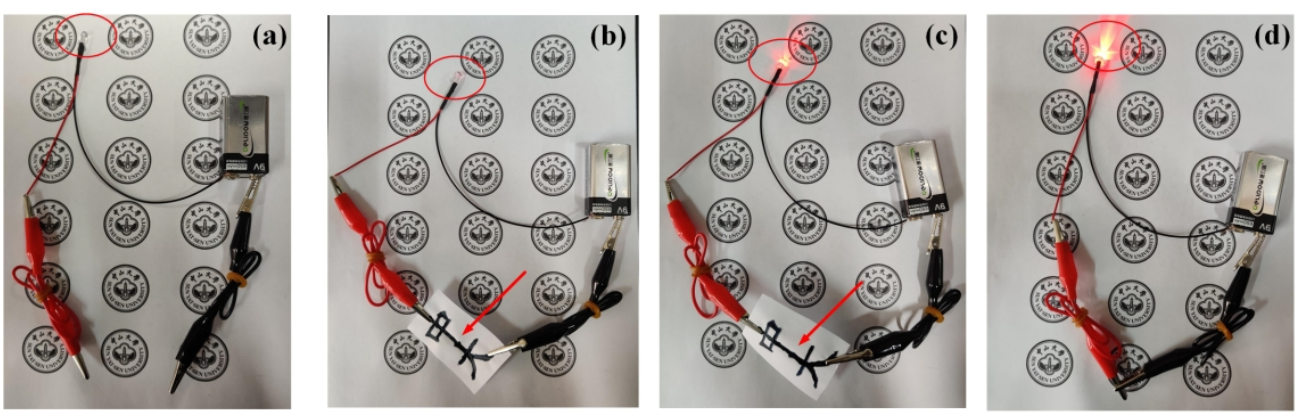

Figure S1. Photo images of LED electrical circuits and the parts for PEDOT:PSS composite deposited on paper are marked with the arrows; (a) Open circuit, (b) Pristine film, (c) PEDOT:PSS/LiTFSI composite, (d) Wire connection only.

$202 \times 101 \mathrm{~mm}(300 \times 300 \mathrm{DPI})$ 

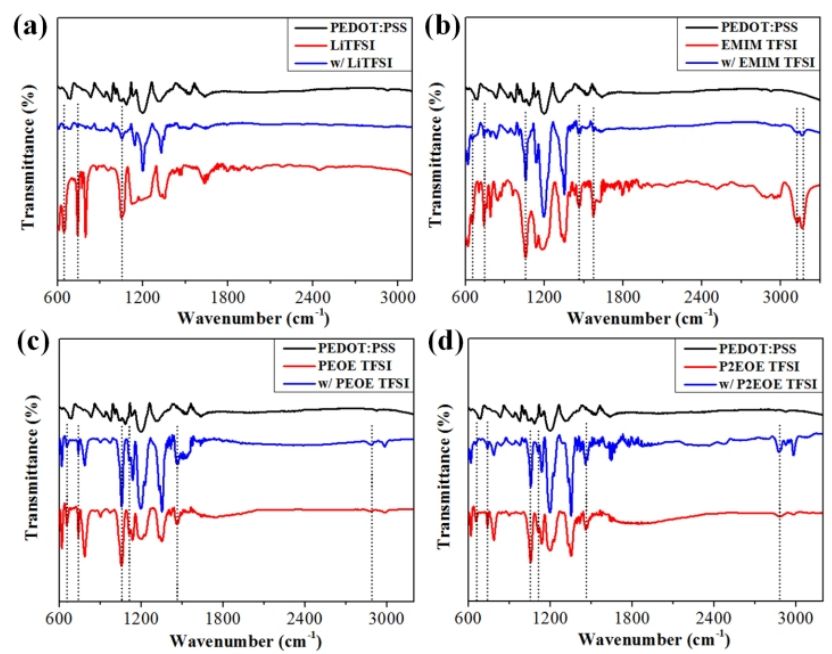

Figure S2. FTIR spectra of pristine film, pure salts and PEDOT:PSS films treated with salts (a) LiTFSI, (b) EMIM TFSI, (c) PEOE TFSI, (d) P2EOE TFSI.

$202 \times 101 \mathrm{~mm}(300 \times 300$ DPI $)$ 


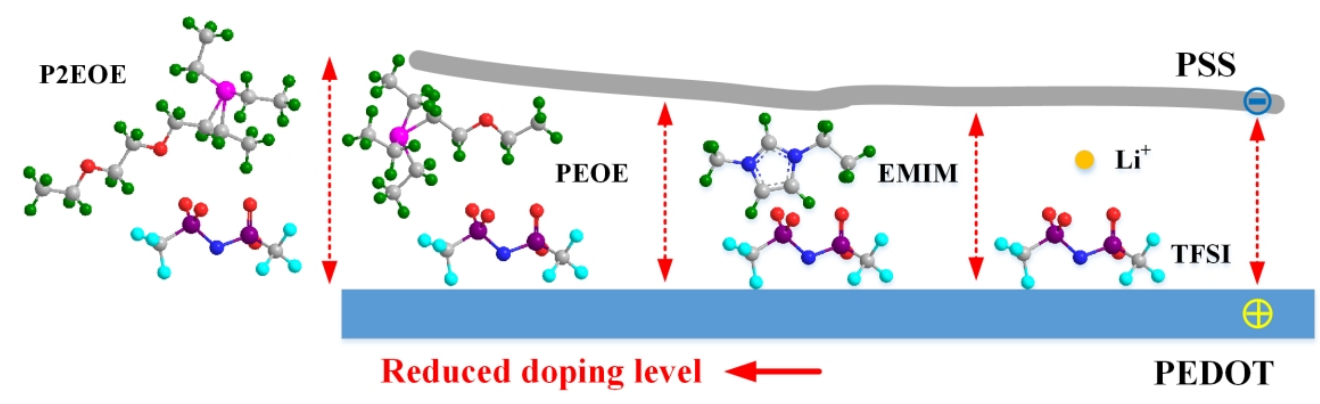

Figure S3. Schematic diagram of cationic size effect of the electrostatic interaction of PEDOT:PSS and salts. $202 \times 101 \mathrm{~mm}(300 \times 300$ DPI $)$ 


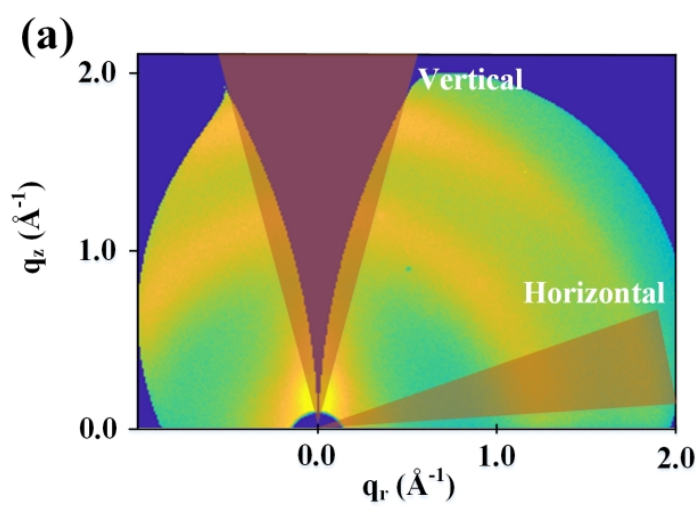

(b)

Figure S4. (a) Exemplary vertical cake cut of $X=-16^{\circ}-16^{\circ}$ and horizontal cake cut of $X=70^{\circ}-85^{\circ}$ on pristine PEDOT:PSS. (b) ח-п stacking distance of horizontal oriented PEDOT crystallites. (c) Apparent crystallite size of horizontal oriented PEDOT.

$202 \times 101 \mathrm{~mm}(300 \times 300 \mathrm{DPI})$ 


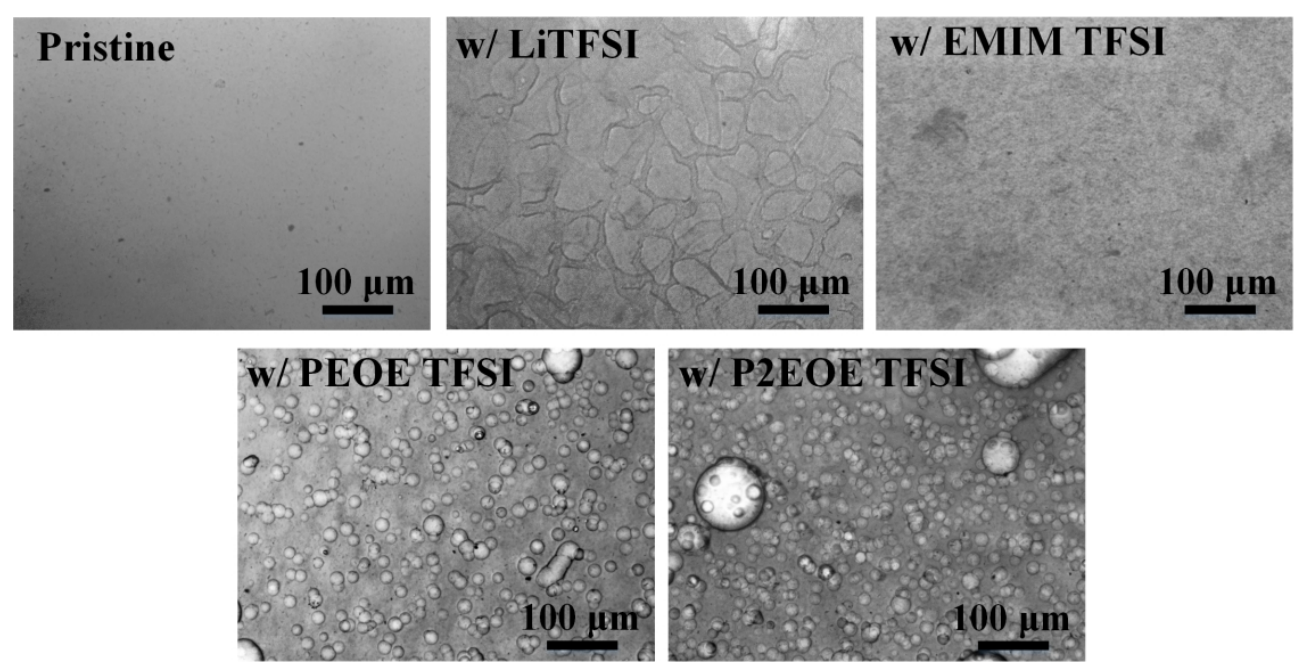

Figure S5. Optical microscope images of PEDOT/PSS films treated with various ionic additives. Note: PEDOT:PSS/salt is denoted as w/salt.

$202 \times 101 \mathrm{~mm}(300 \times 300 \mathrm{DPI})$ 


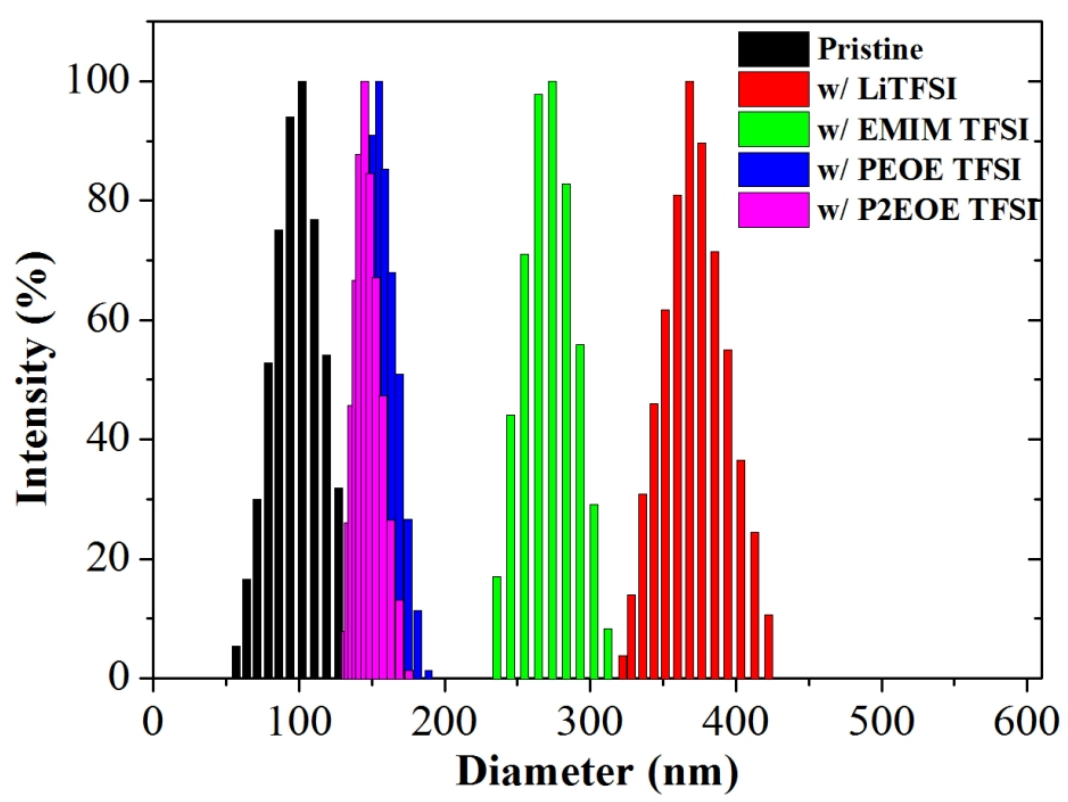

Figure S6. Dynamic light scattering (DLS) data of PEDOT:PSS and salt-mixed PEDOT:PSS dispersions. $127 \times 89 \mathrm{~mm}(300 \times 300 \mathrm{DPI})$ 


\title{
Effects of cationic species in salts on the electrical conductivity of doped PEDOT:PSS films
}

\author{
Xin $\mathrm{Li}^{\mathrm{a}}$, Zhen Liu ${ }^{\mathrm{a}}$, Zekun Zhou ${ }^{\mathrm{a}}$, Haiyang Gao ${ }^{\mathrm{a}}$, Guodong Liang ${ }^{\mathrm{a}}$, Daniel Rauber ${ }^{\mathrm{b}}$, \\ Christopher Kay ${ }^{\mathrm{b}}$, Peng Zhang ${ }^{\mathrm{a},{ }^{*}}$ \\ ${ }^{a}$ School of Materials Science and Engineering, PCFM Lab, Sun Yat-sen University, Guangzhou \\ 510275, China \\ ${ }^{b}$ Department of Chemistry, Saarland University, 66123, Saarbrücken, Germany \\ Corresponding authors: Peng Zhang, E-mail: zhangpeng3@mail.sysu.edu.cn
}

\begin{abstract}
The influence of chemical composition of the doping salts on the conductivity of the poly(3,4-ethylenedioxythiophene):poly(styrenesulfonate) (PEDOT:PSS) films was studied in this work. A series of salts with different cations but the same anion were mixed with PEDOT:PSS. We found out that doping salts of small-size cations led to better conductivity due to the improved crystalline ordering of PEDOT, as revealed by the grazing-incidence wide-angle X-ray scattering (GIWAXS) data. This phenomenon can be rationalized with the fact that small-size cations can dissociate the PSS from PEDOT due to stronger Coulomb interactions, leading to rearrangement of the PEDOT. These findings will help to develop new recipes based on the PEDOT:PSS/salt composite towards the applications for printed flexible electronics, portable displays and flexible energy storage devices.
\end{abstract}

In the past decade, the stretchable flexible electronics have developed fast as essential parts of the popular electronic devices. ${ }^{1-3}$ In these applications, conductive polymers have received tremendous attention as ideal electrodes to replace the traditional indium tin oxide (ITO) ones due to their excellent mechanical properties (i.e., flexibility) and solution 
processability. In particular, poly(3,4-ethylenedioxythiophene):poly(styrenesulfonate) (PEDOT:PSS) is recognized as the most promising candidate owing to its mechanical flexibility, visible-light transmittance, biocompatibility, solution processability and good environmental stability. ${ }^{4-6}$ However, untreated PEDOT:PSS film displays poor electrical conductivity as low as about $1 \mathrm{~S} \mathrm{~cm}^{-1}$, limiting its practical application in popular electronic devices. The low conductivity is mainly attributed to the existence of insulating PSS chains that wrap the conductive PEDOT core and inhibit the formation of PEDOT conductive network. $^{7-9}$ To solve this issue, secondary doping agents like acids, ${ }^{10,11}$ surfactants, ${ }^{12}$ organic solvents ${ }^{13-15}$ and recently the ionic salts ${ }^{16-22}$ are generally introduced into PEDOT:PSS to achieve conductivity that is comparable to the commercial ITO products. Especially the ionic salts in solid or liquid form have garnished particular interest due to their high thermal stability, $^{23}$ unique structural $^{24}$ and electrochemical properties, ${ }^{25}$ as well as an ability to improve conductivity and stretchability of PEDOT:PSS films. ${ }^{20,26}$

Recent studies indicate that ionic interaction between salts and PEDOT:PSS promoted microstructure reorganization which resulted in the formation of PEDOT-rich and PSS-rich microdomains. This eased the formation of interconnected PEDOT networks, resulting in higher electrical conductivities. ${ }^{27-29}$ For example, Badre et al. ${ }^{19}$ reported the conductivity of PEDOT:PSS composite with 1-ethyl-3-methylimidazolium tetracyanoborate (EMIM TCB) was $2084 \mathrm{~S} \mathrm{~cm}^{-1}$ which derived from the salt-induced charge screening between PEDOT and PSS and was three orders of magnitude higher than that of pristine PEDOT:PSS film. Li et al. $^{28}$ revealed ionic exchange between bis(trifluoromethanesulfonyl)imide lithium salt 
(LiTFSI) and PEDOT:PSS (LiTFSI + PEDOT:PSS $\rightleftharpoons$ PEDOT:TFSI + Li:PSS) induced PEDOT microdomain converged together to form a packed and interconnected net structure, which synergistically enhanced the thermoelectric and mechanical performances. Similar ion exchange process and microstructure reorganization was reported by Kee et al. ${ }^{30}$ in the composite of PEDOT:PSS with a series of EMIM cation based ionic liquids (ILs). For an in-depth structural analysis, grazing-incidence wide-angle X-ray scattering (GIWAXS) measurement was employed and disclosed a highly ordered nanofibrillar structures of PEDOT chains that eased the electron transfer. Meanwhile, plenty of recent work have focused on studying the structure-performance relationship in the PEDOT:PSS/salt composites to tune the macroscopic conductivity with molecular designs. It's generally summarized that chemical composition of the salts had strong influence on the conductivity of the PEDOT:PSS/salt composites. Ambroise et al. have claimed that ion exchange (EMIM:anion + PEDOT:PSS $\rightleftharpoons$ EMIM:PSS + PEDOT:anion, i.e., anionic effect) released the PEDOT from the coupling with PSS, which provided the PEDOT higher $p$-doping density and improved conductivity. ${ }^{31}$ However, to the best of our knowledge, the influence of the cations on the structure and conductivity of PEDOT:PSS films have not been explored yet.

In order to investigate the effect of different cationic species on the structure and conductivity of PEDOT:PSS (Orgacon Dry, Agfa Gevaert N.V., Belgium), we chose four salts with various cations, including lithium $\left(\mathrm{Li}^{+}\right)$, 1-ethyl-3-methylimidazolium (EMIM), (2-ethoxyethyl)triethylphosphonium (PEOE) and (2-(2-ethoxyethoxy)ethyl)triethylphosphonium (P2EOE), but the same 
bis(trifluoromethanesulfonyl)amide (TFSI) anion, as demonstrated in Figure 1. For the preparation of PEDOT:PSS/IL composite, $0.05 \mathrm{mmol}$ salt was added into $1000 \mathrm{mg}$ PEDOT:PSS aqueous dispersion (1.6 wt $\%)$, under vigorous stirring.

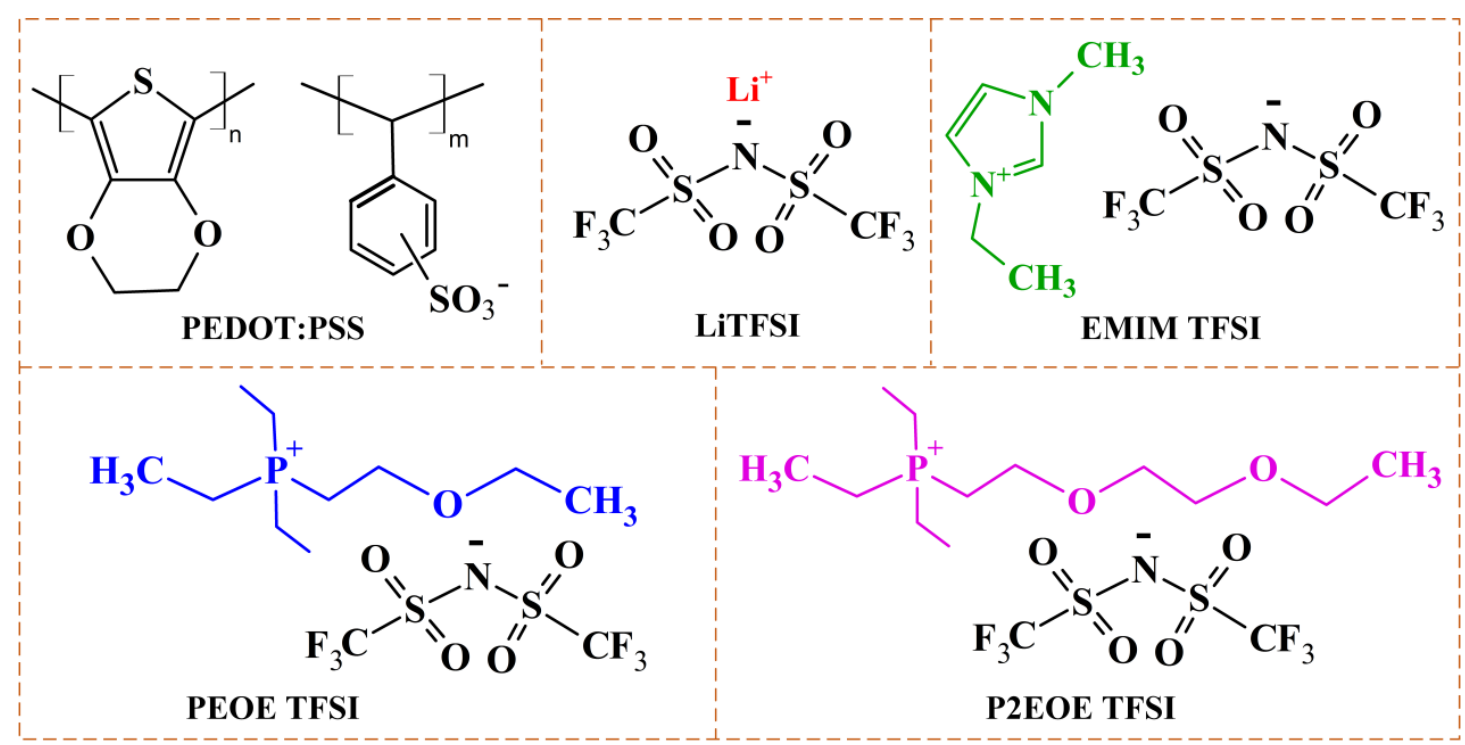

Figure 1. Chemical structure of PEDOT:PSS and ionic salts used in this work.

We chose TFSI as the anion because it was illustrated to be effective for the improvement of the PEDOT conductivity. ${ }^{32}$ The cationic size increases monotonically along this series, $\mathrm{Li}^{+}<\mathrm{EMIM}<\mathrm{PEOE}<\mathrm{P} 2 \mathrm{EOE}$. Meanwhile, these cations were widely used as aqueous electrolyte materials in the previous studies and the self-diffusion coefficients for lithium, EMIM, PEOE and P2EOE cations are $\sim 10 \times 10^{-11}, 33,34 \sim 7 \times 10^{-11}, 352.33 \times 10^{-11}$ and $2.30 \times 10^{-11} \mathrm{~m}^{2} \mathrm{~S}^{-1},{ }^{36}$ respectively. Thus, we can infer that small-size cation showed stronger mobility in PEDOT:PSS aqueous dispersion. The fast mobility of cation helps to combine with PSS anion to form the coupled cation:PSS and screens the Coulomb interaction between PEDOT and PSS. It's expected that salt with small size and high cationic mobility would change significantly the molecular structure and thus the conductivity of PEDOT:PSS/salt in the dried films. 


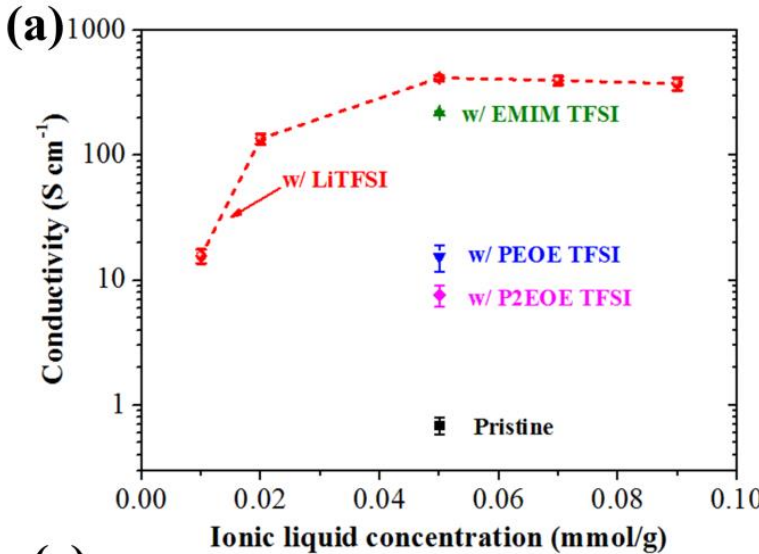

(b)
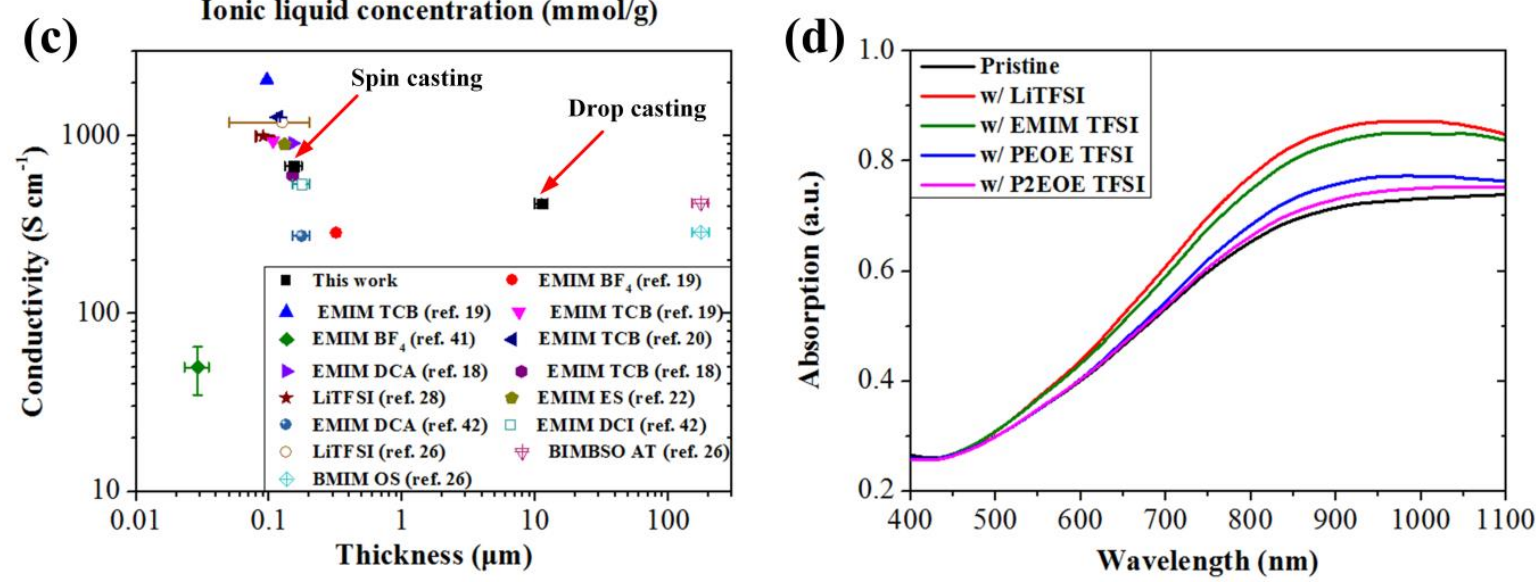

Figure 2. (a) The electrical conductivity result of PEDOT:PSS films doped with different ILs and LiTFSI at a

series of concentration. (b) Photo images of LED electrical circuits and the parts for PEDOT:PSS composite deposited on paper are marked with the arrows (left: PEODT:PSS, right: PEDOT:PSS/LiTFSI composite). (c)

Thickness influence on the conductivity of PEDOT:PSS film (our work is marked by the arrows). (d) UV-Vis absorption spectra of pristine PEDOT:PSS and PEDOT:PSS/IL dispersion. Notes: PEDOT:PSS/salt is denoted as w/salt, $\mathrm{mmol} / \mathrm{g}$ represents the stoichiometry of salts in $1.0 \mathrm{~g}$ of PEDOT: PSS water dispersion.

To verify our hypothesis, we deposited the sample on glass substrate and measured the conductivity of dried films with a multimeter (ST-2258A, Suzhou Jingge Electronic Co., Ltd.) and four-probe method at ca. 58\% relative humidity. By taking the PEDOT:PSS/LiTFSI composite sample as an example, we found that the electrical conductivity of the composite peaked at sample with $0.05 \mathrm{mmol} / \mathrm{g}$ salt (Figure 2a) and this hybrid sample was used in the 
following work. As shown in Figure 2a, the electrical conductivity of PEDOT:PSS films increased after doping with salts. It should be noted that neat salts did not show detectable electrical conductivity. The pristine PEDOT:PSS sample shows a conductivity of $0.83 \mathrm{~S} \mathrm{~cm}^{-1}$, while the conductivity values for polymer composite with $\mathrm{Li}^{+}$, EMIM, PEOE and P2EOE are 418.06, 220.0015 .50 and $7.63 \mathrm{~S} \mathrm{~cm}^{-1}$, respectively (cf. Table $\mathrm{S} 1$ of the supporting information). The enhanced conductivity through mixing with salts is consistent with the previous reports. ${ }^{37-40}$ In addition, there is an obvious difference in the conductivity for the PEDOT:PSS/salt with different cationic components. The smaller-size cations with faster ion diffusivity $\left(\mathrm{Li}^{+}\right.$and EMIM) show better conductivity. Recent work about the mixture of PEDOT:PSS and EMIM TCB has demonstrated that the IL triggered the structural reorganization via ion exchange, i.e., cation:anion + PEDOT:PSS $\rightleftharpoons$ PEDOT:anion + cation:PSS, leading to the formation of highly ordered nanofibrillar structures of PEDOT chains and an enhancement of electrical conductivity. ${ }^{28-30}$ We infer that the small-size cations do ion exchange with PEDOT:PSS that lead to a higher conductivity value in PEDOT:PSS/salt.

To demonstrate the conductivity improvement, we drop casted PEDOT:PSS ink on paper and dried it as a part of the electrical circuit of light-emitting diode (LED), as shown in Figure $2 \mathrm{~b}$ and Figure S1. We found the LED with a PEDOT:PSS/salt circuit was much brighter than the PEDOT:PSS one, confirming the high conductivity of salt-doped PEDOT:PSS and demonstrating its potential application in printed flexible electronics. Moreover, we figured out that the conductivity value of PEDOT:PSS/LiTFSI in this work is comparable to those of 
PEDOT:PSS/salts mentioned in the previous reports, ${ }^{16,18,19,26,41,42}$ see Figure 2c, Table S2 and Table S3 of the supporting information. Note: some deviations are attributed to the sample preparation method and film thickness because our work (Figure 2c) and the others' reports show that spin-casted nanometer films has better conductivity than the drop-casted micrometer ones. $^{26}$

Previous studies indicate that ion exchange reaction between PEDOT:PSS and ILs increased the oxidation state of the PEDOT polymer chains and thus the electronic conductivity. ${ }^{18,29}$ We characterized the oxidation level of PEDOT:PSS with a SHI-MADZU UV-3600 spectrophotometer. As shown in Figure 2d, the broad absorption band near $850 \mathrm{~nm}$ is attributed to oxidation state of PEDOT, i.e., the single polaron with a positive charge. ${ }^{42-44}$ By referring to this peak, we found that PEDOT:PSS/salts films showed a much stronger absorption than PEDOT:PSS, corresponding to an enhanced oxidation level (i.e., higher p-doping density) of PEDOT chains. ${ }^{29}$ The PEDOT conductivity is primarily dependent on the positive charges located along the polymer backbone. ${ }^{39,45}$ Thus the electrical conductivity of PEDOT is proportional to the level of $p$-doping. Indeed, the conductivity descends along the series, PEDOT:PSS/LiTFSI, PEDOT:PSS/EMIM TFSI, PEDOT:PSS/PEOE TFSI, PEDOT:PSS/P2EOE TFSI and pristine PEDOT:PSS, which can be rationalized with a lower $p$-doped level. Furthermore, there is an obvious difference on the $p$-doped level in PEDOT:PSS/salts. The composite with small cations show high-density $p$-doping. This phenomenon is likely derived from the fact that cations with fast mobility are easier to combine with negatively-charged PSS molecules and leave a significant amount of TFSI 

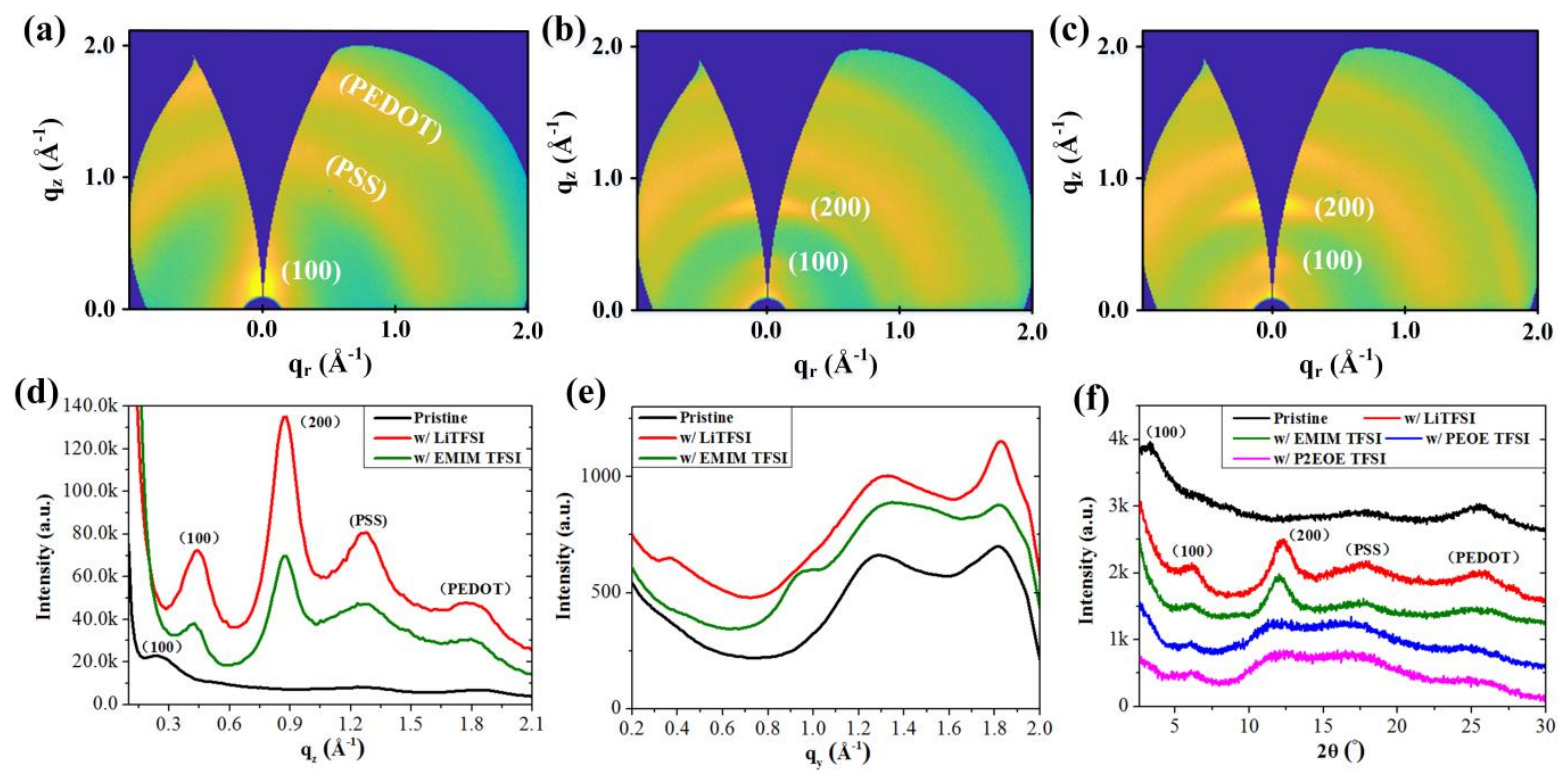

anions for the PEDOT (cf. Figure S2). By referring to the previous reports, ${ }^{31,46}$ we hypothesize that TFSI anions take the role of PSS anions to weaken the screening effects of original PSS anions on PEDOT, thus the oxidized PEDOT ${ }^{+}$cations release from the shielding effect of $\mathrm{PSS}^{-}$and sustain their $p$-doped states. In contrast, the salts with big size and slow cationic mobility such as PEOE and P2EOE could not combine with the $\mathrm{PSS}^{-}$as efficient as that of $\mathrm{Li}^{+}$due to the steric effect (cf. Figure S3).

Figure 3. Typical two-dimensional GIWAXS patterns of (a) pristine, (b) EMIM TFSI-treated and (c) LiTFSI-treated PEDOT:PSS films. (d) Vertical cake cuts of the initial GIWAXS data of pristine and salt-treated PEDOT:PSS films. (e) Horizontal cake cuts of the initial GIWAXS data of pristine and salt-treated PEDOT:PSS films. (f) GIXRD data of pristine and salt-treated PEDOT:PSS films. Note: PEDOT:PSS/salt is denoted as w/salt. In the following, we explore the structure-property relationships of PEDOT:PSS/salt composite with a series of complementary tools. The microstructure of PEDOT:PSS composite films was characterized with GIWAXS based on synchrotron light at 1W1A beamline of BSRF, Beijing, China (cf. supporting information). GIWAXS is a 
well-established protocol to characterize the surface and interface structures of polymer thin film, ${ }^{47-49}$ including PEDOT:PSS before and after IL doping. For example, Kee et al. ${ }^{30}$ demonstrated a highly ordered nanofibrillar structures of PEDOT and Saxena et al. ${ }^{18}$ reported a shortened $\pi-\pi$ stacking distance between PEDOT molecules upon salts' doping. Such structure change leads to an enhanced conductivity of the PEDOT hybrid sample. The sample dispersion was deposited on silicon substrate ((100) orientation, PlutoSemi Co., Ltd) and the thickness of the dried film was around $11 \mu \mathrm{m}$, measured by an Ambios XP-1 profilometer. Figure 3a-c show the typical 2D scattering pattern of GIWAXS. In Figure 3a, three characteristic peaks with $q=0.23 \AA^{-1}(d=27.3 \AA$ calculated with $d=2 \pi / q), 1.26 \AA^{-1}(d=5.0$ $\AA)$, and $1.82 \AA^{-1}(\mathrm{~d}=3.4 \AA)$ are attributed to alternating stacking of PEDOT and PSS (i.e., PEDOT (100) crystal planes), $\pi-\pi$ stacking of PSS, and $\pi-\pi$ stacking of PEDOT, respectively. ${ }^{7,50}$ The lattice spacing distance of $(100)$ plane $\left(\mathrm{d}_{100}\right)$ for pristine PEDOT:PSS is $27.3 \AA$, which decreases to 15.0 and $14.6 \AA$ for PEDOT:PSS/EMIM TFSI and PEDOT:PSS/LiTFSI composite films, respectively (Figure 3d). The decreased $d_{100}$ value means a shorter $\pi-\pi$ stacking distance and improved crystalline ordering along the main chain direction. ${ }^{51}$ Additionally, a new peak corresponding to (200) crystal plane of PEDOT is found at $\mathrm{q}=0.87 \AA^{-1}(\mathrm{~d}=7.2 \AA)$ (Figure 3b-d), indicating an enhanced long-range order of PEDOT crystals after salt doping. ${ }^{52}$ In addition, we studied the in-plane molecular structure of PEDOT:PSS hybrid film by referring to the horizontal cake cuts (Figure 3e) of the initial GIWAXS data. A shortened PEDOT $\pi-\pi$ stacking distance and an improved PEDOT crystallite size in horizontal direction (cf. Figure S4b and S4c) upon the salt doping further 
confirms an enhanced crystalline ordering of PEDOT. ${ }^{52}$ In the PEDOT:PSS composite, carrier mobility depends upon the intermolecular charge transfer rate in the $\pi$ - $\pi$ stacking direction. ${ }^{51,53}$ Namely, more ordered and densely packed PEDOT crystallites in this direction will aid electron transport thereby improve the conductivity. Indeed, the highest conductivity of LiTFSI-treated PEDOT:PSS can be rationalized with the structure changes disclosed by GIWAXS measurements. Similar diffraction results have been reported in highly conductive PEDOT:PSS by Kim et al. ${ }^{8}$ who illustrated that ion exchange between concentrated $\mathrm{H}_{2} \mathrm{SO}_{4}$ and PEDOT:PSS generated a highly ordered and densely packed PEDOT:PSS nanofibril structure. Moreover, the salt-mixing induced conductivity change agrees with the large-scale structure change as observed with optical microscope (cf. Figure S5). In general, LiTFSI-treated PEDOT:PSS shows clear interconnected networks, which might serve as conductive path and ease the electron transport. Thus, we summarize that the well-aligned and interconnected PEDOT domains in the PEDOT:PSS/salt composite resulted in a high conductivity compared to that of the pristine PEDOT:PSS.

To illustrate the generality of the relationship between crystalline structure and conductivity in PEDOT:PSS films mentioned above, the crystalline structure of all four PEDOT:PSS/salt composite films were measured with GIXRD (Bruker D8 Advance, Germany). As shown in the Figure 3f, the curve of pristine PEDOT:PSS film shows three characteristic peaks: $2 \theta=3.3^{\circ}\left(q=0.23 \AA^{-1}\right), 17.7^{\circ}\left(q=1.26 \AA^{-1}\right)$, and $25.8^{\circ}\left(q=1.82 \AA^{-1}\right)$, which are consistent with the GIWAXS data. In addition, the PEDOT:PSS/salt composites show an additional (200) peak and enhanced scattering intensity, like the GIWAXS results. 
Both the GIWAXS and GIXRD results indicate that the improved conductivity of PEDOT:PSS film after doping with salts originates from an enhanced crystalline ordering, agreeing with the previous reports. ${ }^{18,20,42,53}$ We understand the structure change with the facts that the salt anion can play the role of condensing agent for polyelectrolytes such as negatively charged DNA, ${ }^{54}$ i.e., salts hold the $p$-doped PEDOT chains together at a shorter $\pi-$ $\pi$ stacking distance and thus improves the crystalline ordering of PEDOT domains, as demonstrated in Figure 4.

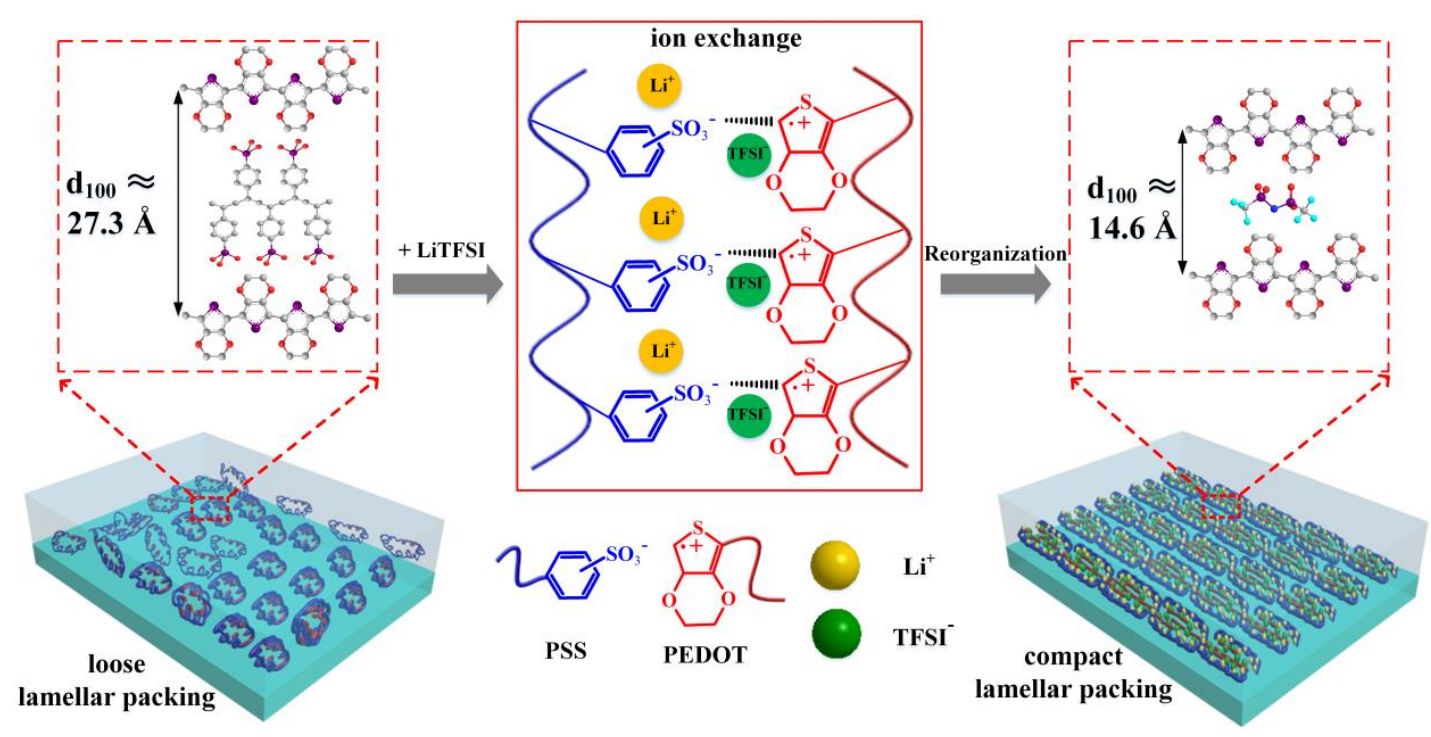

Figure 4. Schematic illustration of the structure of polymer composite film: loose lamellar packing of pristine PEDOT:PSS (left) and compacted lamellar packing of PEDOT:PSS /LiTFSI composite (right).

Figure 4 schematically demonstrates the structure change of PEDOT:PSS film before and after salt doping. In the pristine state, conducting PEDOT molecules are surrounded by insulating PSS molecules to form a stable colloidal dispersion in water ${ }^{26,29}$ and a loose lamellar packing with periodicity of $27.3 \AA$ formed in the dried film. With salt doping, the wrapped PEDOT molecules were released by ion exchange with salt to form a larger colloidal structure in water, as confirmed by the dynamic light scattering data (cf. Figure S6), which 
resulted in a compacted lamellar packing with periodicity of $14.6 \AA$ after solvent evaporation as indicated by the GISAXS and GIXRD data. These structural change as mentioned above explains the 500-fold increase for the conductivity of PEDOT:PSS after doping with LiTFSI.

In summary, we chose four salts with various cations but the same TFSI anion, to study the effect of different cationic species on the structure and conductivity of PEDOT:PSS. The conductivity measurement results revealed that salts with small-size cations endowed the PEDOT:PSS much better conductivity. This phenomenon can be rationalized with the GIWAXS and UV-Vis results: the molecular interactions among salt and PEDOT:PSS made the structural rearrangement of the PEDOT to form ordered crystals and long-range ordered conductive network, as well as the improved $p$-doped level of PEDOT resulted from introducing secondary doping agents. These findings will provide in-depth knowledge about how to manipulate the conductivity of PEDOT:PSS composite towards the applications in printed soft electronics via molecular design and tuning the molecular assembly.

\section{Acknowledgement}

We acknowledge the financial supports from National Natural Science Foundation of China (No. 11905306), Fundamental Research Funds for the Central Universities (No. 19lgpy14) and "100 Top Talents Program" of Sun Yat-sen University. A portion of this work is based on the data obtained at $1 \mathrm{~W} 1 \mathrm{~A}, \mathrm{BSRF}$, China. The authors gratefully acknowledge the technical assistance of Dr. Yu Chen of Diffuse X-ray Scattering Stations during the beamtime.

\section{References}

(1) Gao, M.; Li, L.; Song, Y., Inkjet printing wearable electronic devices. J. Mater. Chem. C 2017, 5 (12), 2971-2993.

(2) Wen, Z.; Yang, Y.; Sun, N.; Li, G.; Liu, Y.; Chen, C.; Shi, J.; Xie, L.; Jiang, H.; Bao, D., A 
wrinkled PEDOT:PSS film based stretchable and transparent triboelectric nanogenerator for wearable energy harvesters and active motion sensors. Adv. Funct. Mater. 2018, 28 (37), 1803684.

(3) Huang, Q.; Zhu, Y., Printing conductive nanomaterials for flexible and stretchable electronics: A review of materials, processes, and applications. Adv. Mater. Technol. 2019, 4 (5), 1800546.

(4) Hui, Y.; Bian, C.; Xia, S.; Tong, J.; Wang, J., Synthesis and electrochemical sensing application of poly (3,4-ethylenedioxythiophene)-based materials: a review. Anal. Chim. Acta 2018, 1022, 1-19.

(5) Kee, S.; Kim, N.; Park, B.; Kim, B. S.; Hong, S.; Lee, J. H.; Jeong, S.; Kim, A.; Jang, S. Y.; Lee, K., Highly deformable and see-through polymer light-emitting diodes with all-conducting-polymer electrodes. Adv. Mater. 2018, 30 (3), 1703437.

(6) Russ, B.; Glaudell, A.; Urban, J. J.; Chabinyc, M. L.; Segalman, R. A., Organic thermoelectric materials for energy harvesting and temperature control. Nat. Rev. Mater. 2016, 1 (10), 1-14.

(7) Palumbiny, C. M.; Liu, F.; Russell, T. P.; Hexemer, A.; Wang, C.; Müller-Buschbaum, P., The crystallization of PEDOT:PSS polymeric electrodes probed in situ during printing. Adv. Mater. 2015, 27 (22), 3391-3397.

(8) Kim, N.; Kee, S.; Lee, S. H.; Lee, B. H.; Kahng, Y. H.; Jo, Y. R.; Kim, B. J.; Lee, K., Highly conductive PEDOT:PSS nanofibrils induced by solution-processed crystallization. Adv. Mater. 2014, 26 (14), 2268-2272.

(9) Lang, U.; Müller, E.; Naujoks, N.; Dual, J., Microscopical investigations of PEDOT:PSS thin films. Adv. Funct. Mater. 2009, 19 (8), 1215-1220.

(10) Mukherjee, S.; Singh, R.; Gopinathan, S.; Murugan, S.; Gawali, S.; Saha, B.; Biswas, J.; Lodha, S.; Kumar, A., Solution-processed poly(3,4-ethylenedioxythiophene) thin films as transparent conductors: Effect of p-toluenesulfonic acid in dimethyl sulfoxide. ACS Appl. Mater. Interfaces 2014, 6 (20), 17792-17803.

(11) Mengistie, D. A.; Ibrahem, M. A.; Wang, P.-C.; Chu, C.-W., Highly conductive PEDOT: PSS treated with formic acid for ITO-free polymer solar cells. ACS Appl. Mater. Interfaces 2014, 6 (4), 2292-2299.

(12) Xia, Y.; Ouyang, J., Highly conductive PEDOT:PSS films prepared through a treatment with geminal diols or amphiphilic fluoro compounds. Org. Electron. 2012, 13 (10), 1785-1792.

(13) Lim, K.; Jung, S.; Lee, S.; Heo, J.; Park, J.; Kang, J.-W.; Kang, Y.-C.; Kim, D.-G., The enhancement of electrical and optical properties of PEDOT:PSS using one-step dynamic etching for flexible application. Org. Electron. 2014, 15 (8), 1849-1855.

(14) Lingstedt, L. V.; Ghittorelli, M.; Lu, H.; Koutsouras, D. A.; Marszalek, T.; Torricelli, F.; Crăciun, N. I.; Gkoupidenis, P.; Blom, P. W., Effect of DMSO Solvent Treatments on the Performance of PEDOT:PSS Based Organic Electrochemical Transistors. Adv. Electron. Mater. 2019, 5 (3), 1800804.

(15) Cui, H.-Q.; Peng, R.-X.; Song, W.; Zhang, J.-F.; Huang, J.-M.; Zhu, L.-Q.; Ge, Z.-Y., Optimization of ethylene glycol doped PEDOT:PSS transparent electrodes for flexible organic solar cells by drop-coating method. Chin. J. Polym. Sci. 2019, 37 (8), 760-766.

(16) Döbbelin, M.; Marcilla, R.; Salsamendi, M.; Pozo-Gonzalo, C.; Carrasco, P. M.; Pomposo, J. A.; Mecerreyes, D., Influence of ionic liquids on the electrical conductivity and morphology of PEDOT:PSS films. Chem. Mat. 2007, 19 (9), 2147-2149.

(17) Armel, V.; Rivnay, J.; Malliaras, G.; Winther-Jensen, B., Unexpected interaction between PEDOT 
and phosphonium ionic liquids. J. Am. Chem. Soc. 2013, 135 (30), 11309-11313.

(18) Saxena, N.; Pretzl, B.; Lamprecht, X.; Bießmann, L.; Yang, D.; Li, N.; Bilko, C.; Bernstorff, S.; Müller-Buschbaum, P., Ionic liquids as post-treatment agents for simultaneous improvement of Seebeck coefficient and electrical conductivity in PEDOT:PSS Films. ACS Appl. Mater. Interfaces 2019, 11 (8), 8060-8071.

(19) Badre, C.; Marquant, L.; Alsayed, A. M.; Hough, L. A., Highly conductive poly (3,4-ethylenedioxythiophene):poly(styrenesulfonate) films using 1-ethyl-3-methylimidazolium tetracyanoborate ionic liquid. Adv. Funct. Mater. 2012, 22 (13), 2723-2727.

(20) Teo, M. Y.; Kim, N.; Kee, S.; Kim, B. S.; Kim, G.; Hong, S.; Jung, S.; Lee, K., Highly stretchable and highly conductive PEDOT:PSS/ionic liquid composite transparent electrodes for solution-processed stretchable electronics. ACS Appl. Mater. Interfaces 2016, 9 (1), 819-826.

(21) Liu, C.; Xu, J.; Lu, B.; Yue, R.; Kong, F., Simultaneous increases in electrical conductivity and Seebeck coefficient of PEDOT:PSS films by adding ionic liquids into a polymer solution. $J$. Electron. Mater. 2012, 41 (4), 639-645.

(22) Teo, M. Y.; RaviChandran, N.; Kim, N.; Kee, S.; Stuart, L.; Aw, K. C.; Stringer, J., Direct patterning of highly conductive PEDOT:PSS/ionic liquid hydrogel via microreactive inkjet printing. ACS Appl. Mater. Interfaces 2019, 11 (40), 37069-37076.

(23) Villanueva, M.; Coronas, A.; García, J.; Salgado, J., Thermal stability of ionic liquids for their application as new absorbents. Ind Eng Chem Res 2013, 52 (45), 15718-15727.

(24) Triolo, A.; Russina, O.; Fazio, B.; Appetecchi, G. B.; Carewska, M.; Passerini, S., Nanoscale organization in piperidinium-based room temperature ionic liquids. J. Chem. Phys. 2009, 130 (16), 164521.

(25) Singh, V. V.; Nigam, A. K.; Batra, A.; Boopathi, M.; Singh, B.; Vijayaraghavan, R., Applications of ionic liquids in electrochemical sensors and biosensors. Int. J. Electrochem. 2012, 2012.

(26) Yue, W.; Zhu, C.; Pfattner, R.; Yan, H.; Jin, L.; Chen, S.; Molinalopez, F.; Lissel, F.; Jia, L.; Rabiah, N. I., A highly stretchable, transparent, and conductive polymer. Sci. Adv. 2017, 3 (3), e1602076.

(27) Luo, J.; Billep, D.; Waechtler, T.; Otto, T.; Toader, M.; Gordan, O.; Sheremet, E.; Martin, J.; Hietschold, M.; Zahn, D. R., Enhancement of the thermoelectric properties of PEDOT:PSS thin films by post-treatment. J. Mater. Chem. A 2013, 1 (26), 7576-7583.

(28) Li, Q.; Deng, M.; Zhang, S.; Zhao, D.; Jiang, Q.; Guo, C.; Zhou, Q.; Liu, W., Synergistic enhancement of thermoelectric and mechanical performances of ionic liquid LiTFSI modulated PEDOT flexible films. J. Mater. Chem. C 2019, 7 (15), 4374-4381.

(29) Li, Q.; Zhou, Q.; Wen, L.; Liu, W., Enhanced thermoelectric performances of flexible PEDOT: PSS film by synergistically tuning the ordering structure and oxidation state. J. Materiomics $\mathbf{2 0 2 0}$, 6, 119-127.

(30) Kee, S.; Kim, N.; Kim, B. S.; Park, S.; Jang, Y. H.; Lee, S. H.; Kim, J.; Kim, J.; Kwon, S.; Lee, K., Controlling molecular ordering in aqueous conducting polymers using ionic liquids. Adv. Mater. 2016, 28 (39), 8625-8631.

(31) de Izarra, A.; Park, S.; Lee, J.; Lansac, Y.; Jang, Y. H., Ionic liquid designed for PEDOT:PSS conductivity enhancement. J. Am. Chem. Soc. 2018, 140 (16), 5375-5384.

(32) Hofmann, A. I.; Katsigiannopoulos, D.; Mumtaz, M.; Petsagkourakis, I.; Pecastaings, G.; Fleury, G.; Schatz, C.; Pavlopoulou, E.; Brochon, C.; Hadziioannou, G., How to choose polyelectrolytes 
for aqueous dispersions of conducting PEDOT complexes. Macromolecules 2017, 50 (5), 1959-1969.

(33) Costa, L. T.; Sun, B.; Jeschull, F.; Brandell, D., Polymer-ionic liquid ternary systems for Li-battery electrolytes: Molecular dynamics studies of LiTFSI in a EMIm-TFSI and PEO blend. $J$. Chem. Phys. 2015, 143 (2), 024904.

(34) Hayamizu, K.; Aihara, Y.; Arai, S.; Martinez, C. G., Pulse-gradient spin-echo ${ }^{1} \mathrm{H}$, ${ }^{7} \mathrm{Li}$, and ${ }^{19} \mathrm{~F}$ NMR diffusion and ionic conductivity measurements of 14 organic electrolytes containing LiN $\left(\mathrm{SO}_{2} \mathrm{CF}_{3}\right)_{2}$. J. Phys. Chem. B 1999, 103 (3), 519-524.

(35) Stolwijk, N.; Obeidi, S., Combined analysis of self-diffusion, conductivity, and viscosity data on room temperature ionic liquids. Electrochim. Acta 2009, 54 (5), 1645-1653.

(36) Philippi, F.; Rauber, D.; Zapp, J.; Hempelmann, R., Transport properties and ionicity of phosphonium ionic liquids. Phys Chem Chem Phys 2017, 19 (34), 23015-23023.

(37) Shi, H.; Liu, C.; Jiang, Q.; Xu, J., Effective approaches to improve the electrical conductivity of PEDOT:PSS: a review. Adv. Electron. Mater. 2015, 1 (4), 1500017.

(38) Kim, G.-H.; Shao, L.; Zhang, K.; Pipe, K. P., Engineered doping of organic semiconductors for enhanced thermoelectric efficiency. Nat. Mater. 2013, 12 (8), 719-723.

(39) Bubnova, O.; Khan, Z. U.; Wang, H.; Braun, S.; Evans, D. R.; Fabretto, M.; Hojati-Talemi, P.; Dagnelund, D.; Arlin, J.-B.; Geerts, Y. H., Semi-metallic polymers. Nat. Mater. 2014, 13 (2), 190-194.

(40) Mazaheripour, A.; Majumdar, S.; Hanemann-Rawlings, D.; Thomas, E. M.; McGuiness, C.; d'Alencon, L.; Chabinyc, M. L.; Segalman, R. A., Tailoring the Seebeck coefficient of PEDOT:PSS by controlling ion stoichiometry in ionic liquid additives. Chem. Mater. 2018, 30 (14), 4816-4822.

(41) Leaf, M. A.; Muthukumar, M., Electrostatic effect on the solution structure and dynamics of PEDOT:PSS. Macromolecules 2016, 49 (11), 4286-4294.

(42) Kee, S.; Kim, H.; Paleti, S. H. K.; El Labban, A.; Neophytou, M.; Emwas, A.-H.; Alshareef, H. N.; Baran, D., Highly stretchable and air-stable PEDOT:PSS/ionic liquid composites for efficient organic thermoelectrics. Chem. Mater. 2019, 31 (9), 3519-3526.

(43) Massonnet, N.; Carella, A.; Jaudouin, O.; Rannou, P.; Laval, G.; Celle, C.; Simonato, J.-P., Improvement of the Seebeck coefficient of PEDOT:PSS by chemical reduction combined with a novel method for its transfer using free-standing thin films. J. Mater. Chem. C 2014, 2 (7), 1278-1283.

(44) Lee, J.-g.; Cho, W.; Kim, Y.; Cho, H.; Lee, H.; Kim, J. H., Formation of a conductive overcoating layer based on hybrid composites to improve the stability of flexible transparent conductive films. RSC Adv. 2019, 9 (8), 4428-4434.

(45) Fabretto, M. V.; Evans, D. R.; Mueller, M.; Zuber, K.; Hojati-Talemi, P.; Short, R. D.; Wallace, G. G.; Murphy, P. J., Polymeric material with metal-like conductivity for next generation organic electronic devices. Chem. Mater. 2012, 24 (20), 3998-4003.

(46) Brooke, R.; Fabretto, M.; Krasowska, M.; Talemi, P.; Pering, S.; Murphy, P. J.; Evans, D., Organic energy devices from ionic liquids and conducting polymers. J. Mater. Chem. C 2016, 4 (7), 1550-1556.

(47) Zhang, P.; Rothkirch, A.; Koch, M.; Roth, S.; Kraus, T., Determination of the surface facets of gold nanorods in wet-coated thin films with grazing-incidence wide angle X-ray scattering. Part 
Part Syst Char 2019, 36 (12), 1900323.

(48) Zhang, P.; Huang, H.-y.; Chen, Y.; Yu, S.; Krywka, C.; Vayalil, S. K.; Roth, S. V.; He, T.-b., Preparation of long-range ordered nanostructures in semicrystalline diblock copolymer thin films using micromolding. Chin. J. Polym. Sci. 2014, 32 (9), 1188-1198.

(49) Müller-Buschbaum, P., The active layer morphology of organic solar cells probed with grazing incidence scattering techniques. Adv. Mater. 2014, 26 (46), 7692-7709.

(50) Kim, N.; Lee, B. H.; Choi, D.; Kim, G.; Kim, H.; Kim, J.-R.; Lee, J.; Kahng, Y. H.; Lee, K., Role of interchain coupling in the metallic state of conducting polymers. Phys Rev Lett 2012, 109 (10), 106405 .

(51) Rivnay, J.; Noriega, R.; Northrup, J. E.; Kline, R. J.; Toney, M. F.; Salleo, A., Structural origin of gap states in semicrystalline polymers and the implications for charge transport. Phys Rev B 2011, 83 (12), 121306.

(52) Bießmann, L.; Saxena, N.; Hohn, N.; Hossain, M. A.; Veinot, J. G.; Müller-Buschbaum, P., Highly Conducting, Transparent PEDOT: PSS polymer electrodes from post-treatment with weak and strong acids. Adv. Electron. Mater. 2019, 5 (2), 1800654.

(53) Petsagkourakis, I.; Pavlopoulou, E.; Portale, G.; Kuropatwa, B. A.; Dilhaire, S.; Fleury, G.; Hadziioannou, G., Structurally-driven enhancement of thermoelectric properties within poly(3,4-ethylenedioxythiophene) thin films. Sci. Rep. 2016, 6, 30501.

(54) Lansac, Y.; Degrouard, J.; Renouard, M.; Toma, A. C.; Livolant, F.; Raspaud, E., A route to self-assemble suspended DNA nano-complexes. Sci. Rep. 2016, 6, 21995. 